\title{
Establishment and Evaluation of Bone Mineral Density Reference Databases for Multiple Skeletal Regions in Women in Shandong's Coastal Areas
}

\author{
Qiuling Wang ${ }^{1 \#}$, Huafeng Li ${ }^{1 \#}$, Weiwei Xiao" ${ }^{2 \#}$, Zhenying Liu${ }^{1 \#}$, Guiqin Gu${ }^{1 \#}$, Jinhuan Wang ${ }^{1}$, Yuan $\mathrm{Hu}^{3}$, \\ Shulin Chen ${ }^{1}$, Qingjie Xue ${ }^{2,3 *}$ and Daping Wang ${ }^{1 *}$ \\ ${ }^{1}$ Department of Endocrinology, Affiliated Yantai Yuhuangding Hospital of Qingdao University, university, Yantai, Shandong, 264000 \\ PR China \\ ${ }^{2}$ Basic Medical School, Jining Medical University, Shandong, PR China
}

${ }^{3}$ Institute of Immunology, Icahn School of Medicine at Mount Sinai, New York, NY 10029, USA

${ }^{\#}$ These authors contributed equally to this paper

*Corresponding author: Qingjie Xie, Institute of Immunology, Icahn School of Medicine at Mount Sinai, Basic Medical School,

Shandong, PR China

Daping Wang, Department of Endocrinology, Affiliated Yantai Yuhuangding Hospital of Qingdao University, Yantai, Shandong, China

\section{ARTICLE INFO}

Received: May 17, 2019

Published: 蔧 May 23, 2019

Citation: Qiuling W, Huafeng L, Weiwei X, Zhenying L, Guiqin G, Jinhuan W, Yuan H, Shulin C, Qingjie X, Daping W. Establishment and Evaluation of Bone Mineral Density Reference Databases for Multiple Skeletal Regions in Women in Shandong's Coastal Areas. Biomed J Sci \& Tech Res 18(2)-2019. BJSTR. MS.ID.003129.

Keywords: BMD; Calibration; Reference Databases; Women; OP
ABSTRACT

Objective: The aim of our research was to establish and evaluate bone mineral density (BMD)reference databases in women in Shandong's coastal areas, that would be useful for diagnosis of osteoporosis(OP).

Methods: Thirty healthy women aged 25-35 years were selected and had their BMD measured respectively by two kinds of dual-energy X-ray absorption(DXA, Lunnar i, USA and Challenger, France) at skeletal regions of lumbar spine $\left(\mathrm{L}_{2}, \mathrm{~L}_{3}\right.$ and $\left.\mathrm{L}_{4}\right)$, left hip( femoral neck), and established the calibration equations between them. Compared BMD of women in Qingdao and Yantai which was calibrated before and after, eight kinds of regression models were fitted with age-related change. The best fitting model equations were found, and the reference database was established. Prevalence of osteopenia and OP were analyzed according to these databases.

Results: BMD of Challenger and Lunar i DXAs showed significant linear relationship. BMD after calibration in 4 skeletal regions changed with aging, and a cubic regression model was better fitted with aged-related change as compared with other regression models. BMD reference databases of women in Shandong's coastal areas were established by cubic regression equation. The peak BMD appeared at 20-40 years old and followed by a linear decreasing after 40 years old. Females who had medical examination from 2010 to 2017, aged 50 to 89 were studied by this database. It found that the prevalence of OP increased with age and lower than other cities. The morbidity of OP based on the database of Challenger DXA was more than that our database of Shandong's coastal areas. Conclusion: This study established the BMD reference databases for multiple skeletal regions of women in Shandong's coastal areas that was the reliable reference standard for diagnosing of OP.

\section{Introduction}

BMD, which is an important symbol of bone quality, can establish the diagnosis of OP before the first fracture has occurred $[1,2]$. BMD is affected by many factors, such as races, lifestyles, customs, territory, etc. Qingdao and Yantai two large coastal cities in Shandong province, are close to each other. They have the similar latitude region, the amount of sunlight, lifestyle, dietary habits, diet structure, cultural background, dietary calcium intake and no difference of ethnic distribution. Therefore, there was no 
significant difference of BMD between the two district residents. However, Bone mineral density instruments are different in these two cities ( Challenger DXAs in Qingdao and Lunar i in Yantai), and the different kinds of DXAs are the important factors, which may influence the detecting results of BMD. Hence, their BMD results or data are not comparable before calibration [3]. It must be calibration between different kinds of DXAs in order to keep the consistency of the results. It was reported in European to calibrate results of different kinds of DXAs using European spin phanton and one hundred healthy females at 20-80 years old which was costly and tedious work [4]. Chinese researches used body models or anthropomorphic lumbar spine models for this purpose. This measurement cannot cover the operating error of technician (such as position, interesting region) [5]. In our research, peak bone mass people were selected to calibrate between Lunar i and Challenger DXAs, that covered not only the instrument precision but also technicians operating errors and reflected the diagnostic value of OP. Through the calibration of different DXAs, the aim of this research was to establish the BMD reference database in Shandong's coastal areas, then we identified the rationality and practicality by comparing BMD databases differences of Yantai and Qingdao and layed the foundation for accurate diagnosis and clinical observation of osteoporosis(OP).

\section{Results}

\section{Cross-Calibration of Two Types of DXAs}

The datas of BMD at the lumbar spine $\left(\mathrm{L}_{2}-\mathrm{L}_{4}\right)$ and left hip (femoral neck) which were measured by Challenger and Lunar i DXAs respectively for thirty females were shown in Table 1. The results of DXAs (Lunar i in Yantai) were more than those of DXA(Challenger in Qingdao). It indicated that BMD data measured by different types of DXAs must correct before comparing them.

Table 1: BMD of healthy females obtained from Challenger and Lunar i DXAs $\left(\mathrm{g} / \mathrm{cm}^{2}, \bar{x} \pm s, \mathrm{n}=30\right)$.

\begin{tabular}{|c|c|c|c|c|}
\hline DXA & L2 & L3 & L4 & Femoral neck \\
\hline Challenger & $1.214 \pm 0.134^{*}$ & $1.341 \pm 0.140^{*}$ & $1.285 \pm 0.147^{*}$ & $1.002 \pm 0.114^{*}$ \\
\hline Lunar i & $1.021 \pm 0.122$ & $1.051 \pm 0.124$ & $0.996 \pm 0.126$ & $0.895 \pm 0.112$ \\
\hline
\end{tabular}

Note: ${ }^{*}, P<0.05$, Challenger vs. Lunar i.

The regression equations and related coefficients of BMD at different bone regions are below. It's shown that there was a significant linear correlation between the data of BMD by two kinds of DXAs.

$$
\begin{aligned}
& \mathrm{L}_{2}: \mathrm{BMD}_{\text {Challenger }}=1.315 \mathrm{BMD}_{\text {Lunar i }}-0.126, \quad \mathrm{r}=0.926 \\
& \mathrm{~L}_{3}: \mathrm{BMD}_{\text {Challenger }}=1.669 \mathrm{BMD}_{\text {Lunar i }}-0.408, \quad \mathrm{r}=0.904 \\
& \mathrm{~L}_{4}: \mathrm{BMD}_{\text {Challenger }}=1.565 \mathrm{BMD}_{\text {Lunar } \mathrm{i}}-0.271, \quad \mathrm{r}=0.922
\end{aligned}
$$
0.957

Femoral neck : $\mathrm{BMD}_{\text {Challenger }}=1.267 \mathrm{BMD}$ Lunar i $-0.132, \mathrm{r}=$

\section{Comparison of BMD Reference Databases Between Yan- tai and Qingdao}

BMD of healthy women of Yantai and Qingdao at the same bone area before cross-calibration were different significantly $(\mathrm{P}<0.01)$ in Table 2. BMD datas at the same bone area and age after crosscalibration were no significant difference $(\mathrm{P}>0.05)$. The results were shown in Table 3. It showed that BMD data decreased gradually with the increasing age and had the rising trend at about 35 years old. It continued decreasing after 35 years old and decreased dramatically after 45 years old.

Table 2: BMD of health women from Yantai before cross-calibration $\left(\mathrm{g} / \mathrm{cm}^{2}, x \pm s\right)$.

\begin{tabular}{|c|c|c|c|c|c|}
\hline Age & $\mathbf{n}$ & $\mathbf{L}_{2}$ & $\mathbf{L}_{3}$ & $\mathbf{L}_{4}$ & Femoral Neck \\
\hline $20-$ & 23 & $1.008 \pm 0.096^{*}$ & $1.014 \pm 0.068$ & $0.947 \pm 0.077^{*}$ & $0.891 \pm 0.074^{*}$ \\
\hline $25-$ & 69 & $1.011 \pm 0.113$ & $1.001 \pm 0.092^{*}$ & $0.955 \pm 0.105^{*}$ & $0.856 \pm 0.099^{*}$ \\
\hline $30-$ & 48 & $0.985 \pm 0.101^{*}$ & $0.994 \pm 0.081$ & $0.962 \pm 0.105$ & $0.845 \pm 0.085$ \\
\hline $35-$ & 75 & $1.004 \pm 0.113$ & $1.006 \pm 0.093^{*}$ & $0.987 \pm 0.102^{*}$ & $0.859 \pm 0.097^{*}$ \\
\hline $40-$ & 162 & $0.981 \pm 0.105^{*}$ & $0.993 \pm 0.086^{*}$ & $0.951 \pm 0.099$ & $0.840 \pm 0.108^{*}$ \\
\hline $45-$ & 257 & $0.982 \pm 0.117^{*}$ & $0.991 \pm 0.095$ & $0.960 \pm 0.112^{*}$ & $0.838 \pm 0.085^{*}$ \\
\hline $50-$ & 361 & $0.934 \pm 0.118^{*}$ & $0.957 \pm 0.096$ & $0.935 \pm 0.109$ & $0.825 \pm 0.099^{*}$ \\
\hline $55-$ & 310 & $0.895 \pm 0.104^{*}$ & $0.924 \pm 0.090^{*}$ & $0.900 \pm 0.106$ & $0.804 \pm 0.091^{*}$ \\
\hline $60-$ & 358 & $0.865 \pm 0.102$ & $0.904 \pm 0.088^{*}$ & $0.891 \pm 0.103$ & $0.782 \pm 0.083^{*}$ \\
\hline $65-$ & 257 & $0.856 \pm 0.118^{*}$ & $0.907 \pm 0.100^{*}$ & $0.895 \pm 0.120$ & $0.770 \pm 0.091$ \\
\hline $70-$ & 180 & $0.863 \pm 0.098^{*}$ & $0.906 \pm 0.093^{*}$ & $0.905 \pm 0.104^{*}$ & $0.753 \pm 0.085^{*}$ \\
\hline $75-$ & 108 & $0.850 \pm 0.115^{*}$ & $0.887 \pm 0.093$ & $0.790 \pm 0.098^{*}$ & $0.713 \pm 0.062^{*}$ \\
\hline $80-$ & 66 & $0.834 \pm 0.139$ & $0.873 \pm 0.109^{*}$ & $0.781 \pm 0.068^{*}$ & $0.688 \pm 0.071^{*}$ \\
\hline
\end{tabular}

Note: *, $\mathrm{P}<0.01$, vs. Qingdao females [6]. 
Table 3: BMD of health women from Yantai after cross-calibration $\left(\mathrm{g} / \mathrm{cm}^{2}, x \pm s\right)$.

\begin{tabular}{|c|c|c|c|c|c|}
\hline Age & $\mathbf{n}$ & $\mathbf{L}_{2}$ & $\mathbf{L}_{3}$ & $\mathbf{L}_{4}$ & Femoral Neck \\
\hline $20-$ & 23 & $1.199 \pm 0.128$ & $1.285 \pm 0.114$ & $1.211 \pm 0.121$ & $0.996 \pm 0.092$ \\
\hline $25-$ & 69 & $1.203 \pm 0.151$ & $1.263 \pm 0.155$ & $1.224 \pm 0.165$ & $0.953 \pm 0.125$ \\
\hline $30-$ & 48 & $1.168 \pm 0.131$ & $1.251 \pm 0.137$ & $1.233 \pm 0.165$ & $0.939 \pm 0.108$ \\
\hline $35-$ & 162 & $1.194 \pm 0.150$ & $1.271 \pm 0.156$ & $1.273 \pm 0.161$ & $0.960 \pm 0.123$ \\
\hline $40-$ & 75 & $1.164 \pm 0.137$ & $1.249 \pm 0.146$ & $1.217 \pm 0.155$ & $0.932 \pm 0.136$ \\
\hline $45-$ & 257 & $1.165 \pm 0.152$ & $1.246 \pm 0.161$ & $1.233 \pm 0.175$ & $0.930 \pm 0.108$ \\
\hline $50-$ & 361 & $1.102 \pm 0.157$ & $1.189 \pm 0.161$ & $1.192 \pm 0.171$ & $0.913 \pm 0.124$ \\
\hline $55-$ & 310 & $1.051 \pm 0.136$ & $1.134 \pm 0.151$ & $1.136 \pm 0.166$ & $0.887 \pm 0.116$ \\
\hline $60-$ & 358 & $1.011 \pm 0.134$ & $1.103 \pm 0.147$ & $1.121 \pm 0.163$ & $0.859 \pm 0.104$ \\
\hline $65-$ & 257 & $1.001 \pm 0.156$ & $1.106 \pm 0.166$ & $1.128 \pm 0.188$ & $0.844 \pm 0.116$ \\
\hline $70-$ & 180 & $1.009 \pm 0.131$ & $1.107 \pm 0.155$ & $1.143 \pm 0.166$ & $0.822 \pm 0.108$ \\
\hline $75-$ & 108 & $0.992 \pm 0.152$ & $1.072 \pm 0.155$ & $0.965 \pm 0.152$ & $0.771 \pm 0.076$ \\
\hline $80-$ & 66 & $0.971 \pm 0.183$ & $1.049 \pm 0.183$ & $0.952 \pm 0.106$ & $0.740 \pm 0.091$ \\
\hline
\end{tabular}

Establishment of BMD Reference Databases in regression models (linear regression, logistic regression, quadric

\section{Shandong's Coastal Areas}

The BMD of Yantai and Qingdao had divided into groups by every 5 years old and excluded the ones less or more than 3SD of average. BMD of 4 skeletal regions with age-related changes were fitted in the whole healthy female population by eight kinds of regression, cubic regression, composite regression, exponential regression, growth regression and exponential regression model). The results indicated that the cubic regression model was better as compared to the others, and namely which of the coefficients of determination $\left(R^{2}\right)$ was always the biggest. It was shown that $R^{2}$ of $\mathrm{L}_{4}$ (lumbar vertebrae) was the minimum (0.3557) in Figure 1.
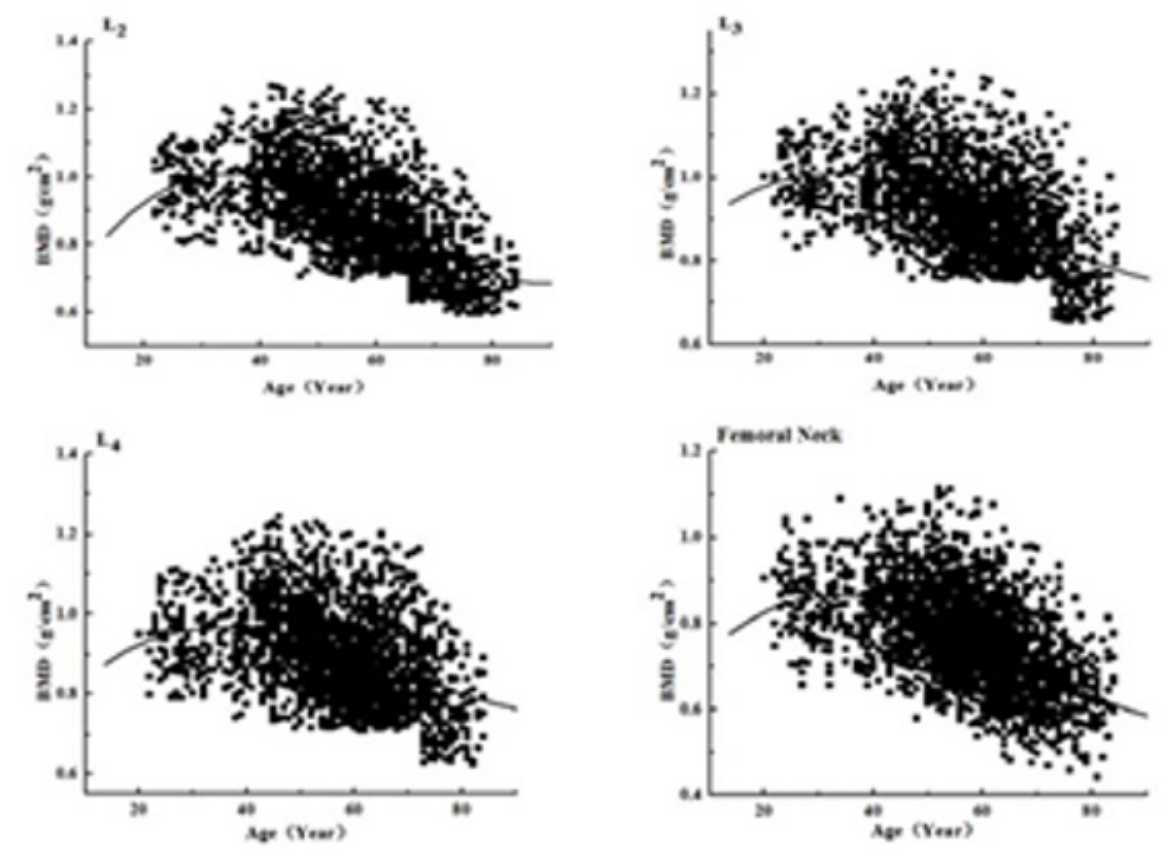

Figure 1: Scatter plot and cubic regression curve of age-related change of BMD at various skeletal regions

$$
\begin{array}{cc}
L 2: R^{2}=0.6028 P<0.01 & L 3: R^{2}=0.4125 P<0.01 \\
y=0.4847+0.02186 x-0.0005061 x^{2}+0.000002890 x^{3} \quad y=0.4037+0.01762 x-0.0003209 x^{2}+0.000001582 x^{3} \\
L 4: R^{2}=0.5726 P<0.0 \quad \text { Femoral Neck }: R^{2}=0.4726 P<0.01 \\
y=0.3333+0.04512 x-0.0009143 x^{2}+0.000005152 x^{3} \quad y=0.7661+0.0684 x-0.0003436 x^{2}+0.000001726 x^{3}
\end{array}
$$




\section{Morbidity of OP}

The diagnostic standard of osteopenia and OP are defined by the World Health Organization (WHO) for women aged over 50 years [7]. The morbidity of OP at 4 skeletal regions were detected respectively by using BMD reference databases established in our study and self-provided database by Challenger in Table 4 . The results showed that the morbidity of OP at lumbar spine $\left(\mathrm{L}_{2}-\mathrm{L}_{4}\right)$ and the left hip (femoral neck) gradually increased with increasing age $\left(\mathrm{L}_{2}\right.$ from $10.6 \%$ to $32.5 \%$; $\mathrm{L} 3$ from $7.7 \%$ to $25.3 \%$; $\mathrm{L} 4$ from $7.9 \%$ to $17.9 \%$; Femoral Neck from $1.5 \%$ to $34.3 \%$;). And they also showed that the morbidity of OP at lumbar spine $\left(\mathrm{L}_{2}-\mathrm{L}_{4}\right)$ and the left hip (femoral neck) by the database of Challenger DXA were more than that by our database of shandong's coastal areas, Which have significant difference $(\mathrm{P}<0.05)$, at the same time, the difference was greater in the group aged $80-89$ years $(\mathrm{P}<0.01)$.

Table 4: Two databases comparison of morbidity of OP: established in our study (self-provided) (\%).

\begin{tabular}{|c|c|c|c|c|}
\hline Skeletal Regions & $50-59$ & $60-69$ & 70-79 & 80-89 \\
\hline $\mathrm{L}_{2}$ & $10.6(18.6)^{*}$ & $16.6(20.1)^{*}$ & $28.3(36.4)^{*}$ & $36.5(58.9)^{* *}$ \\
\hline $\mathrm{L}_{3}$ & $7.7(18.3)^{*}$ & $14.5(16.1)$ & $24.4(34.6)^{*}$ & $25.3(56.7)^{* *}$ \\
\hline $\mathrm{L}_{4}$ & $7.9(13.8)^{*}$ & $10.8(12.7)^{*}$ & $17.5(33.9)^{*}$ & $17.9(36.2)^{* *}$ \\
\hline Femoral Neck & $1.5(9.2)^{*}$ & $25.6(29.6)$ & $23.4(47.8)^{*}$ & $34.3(58.7)^{* *}$ \\
\hline
\end{tabular}

Note: ${ }^{*}<<0.05,{ }^{* *} \mathrm{P}<0.01$ compared with self-provided BMD databases.

\section{Discussion}

The kinds of DXA in the world mainly have the Lunar, Hologic, Norland of America and Medilink, Challenger of French. The correction formula of the first three DXAs(Lunar, Hologic, Norland) had been established and widely used. However, there was no report about French DXA of Challenger and Medilink. This research aimed to establish the data conversion relationship of DXAs (Challenger in Qingdao district and Lunar i in Yantai district), which made it possible for these DXAs data shared. We selected 30 healthy women aged from 25 to 35 years old to scan respectively in the Challenger and Lunar i DXA, the measured data was used to do regression analysis and calculate the correction formula. The results showed the correlation coefficients of all skeletal regions were above 0.9 , which indicated the obvious correlation. The bone metabolism is affected by heredity, race, region, lifestyle, etc. There was no significant difference of BMD between peer group of the same ethnic and geographical environment $[8,9]$, while the BMD datas from different races cannot be shared. Recently almost all the laboratory used "Oriental" reference databases which were configured by imported instrument factory to diagnose OP, and the results of the accuracy and reliability were affected by them [10]. This research report provided BMD reference range in the shandong's coastal areas. This research selected 6982 healthy women including 3274 Yantai's people and 3708 Qingdao's people as samples, and they came from different districts and county-level cities. Because Yantai and Qingdao have the similar latitude region, the amount of sunlight, lifestyle, dietary habits, diet structure, cultural background, dietary calcium intake, and no difference of ethnic distribution, these factors determined there was no significant difference of BMD data to establish the database and it can be applied to the shandong's coastal areas. The research found that all peak bone mass of BMD occurred between the ages of 2040 years old and the linear decrease began after 40 years. The main reason might be because estrogen levels of middle-aged and old women decline, especially after entering the menopause, lead to bone mass loss seriously and then BMD decrease $[11,12]$.
The morbidity of OP and osteopenia at lumbar spine $\left(\mathrm{L}_{2}-\mathrm{L}_{4}\right)$ and the left hip (femoral neck) were studied. The results showed that the prevalence of OP increased from 1.5-10.6\% (40-59 years old) to 17.9-34.3\% (80-89 years old). Tongqian zhang et al. [13] discussed senior citizens suffered from OP in Lanzhou area to find that OP incidence reduced more than $80 \%$ after 70 years. Wenzhi wang et al. [14] analyzed the prevalence rate of OP in middle-aged and aged people in Chengdu. The results showed that the OP incidence of lumbar spine and hip had basically reached $90 \%$ after 70 years and $100 \%$ after the age of 80 years. Zhihai zhang et al. [15] surveyed aged-related prevalence of OP in Chinese women and the results showed that prevalence of OP was $21.75 \pm 5.96 \%$ after 50 years, and $76.74 \pm 5.28$ at the age of $80-90$. Above all, the prevalence of OP in the coastal areas of shandong was lower than other domestic areas significantly, the main reason is that diet of coastal areas have a large number of seafood, which are rich in calcium. Calcium is important for building materials of bone. Meanwhile, the research compared the database of shandong's coastal areas which established in our study with the Challenger DXA self-provided database and used them to diagnose the detection rate of OP in women of Yantai and Qingdao. The results showed that the morbidity of OP at lumbar spine $\left(\mathrm{L}_{2}-\mathrm{L}_{4}\right)$ and the left hip (femoral neck) by the database of Challenger DXA were more than that by the database of shandong's coastal areas. The reason may be the local diet which has the richer calcium in sea food and it made the local peak BMD higher than that of Challenger DXA self-provided. Considering the morbidity of OP by the reference databases in our study in group aged 8089 years old the difference was greater than any other groups, the reason is that of the amount of sunlight, lifestyle, dietary habits, diet structure, cultural background, dietary calcium intake than any other district. It's said that the Challenger DXA self-provided database doesn't apply to women of the coastal area. Through the research on the incidence of OP and osteopenia, it can be seen that BMD reference databases for multiple skeletal regions in women in Shandong's coastal areas has strong applicability. 


\section{Materials and Methods}

\section{Subjects}

All subjects were ruled out conditions or medications known to affect bone metabolism (such as chronic renal failure, hyperthyroidism and hyperparathyroidism, diabetes, various cancers, oophorectomy, those received diuretics, glucocorticoid and Calcium medicinal preparation). It excluded special occupation persons (such as athletes, the basement staff, etc.) and those staying in bed for three months. Thirty healthy women at 25-35 years old were selected and had their BMD measured respectively by two kinds of DXAs (Challenger, France and Lunar i, USA) at skeletal regions of lumbar spine $\left(L_{2}, L_{3}\right.$ and $\left.L_{4}\right)$, left hip ( femoral neck). All subjects signed the written informed consents. 3274 citizens aged from 20 to 84 years old who come from Yantai were screened to establish BMD databases by Lunar i DXAs at Yuhuangding Hospital of Yantai from 2010 to 2017. BMD databases of 3708 citizens who came from Qingdao scanned by Challenger DXA at Haici Hospital of Qingdao had been established [5].

\section{BMD Measurements}

When measuring BMD each subject was positioned supine, and the physiological lumbar lordosis was flattened by elevation of the knees. Measurements in one city were performed and analyzed by the same person. Calibration and quality control were performed on a daily basis according to manufacturer's recommendation.

World Health Organization criteria [16] was used to categorize the scans as follows: T-score below or equal -2.5 indicated $\mathrm{OP}$; between -2.5 and -1 , osteopenia; and equal or greater than -1 , normal bone density. T-scores were derived using the manufacturer's local population reference database. The coefficient of variation for the precision of BMD measurements at all sites was less than $1 \%$

\section{Statistical Analysis}

All the data were analyzed with the SPSS 13.0. The results were expressed as Mean \pm SD. The comparison between two groups was conducted with the $t$ test, a value of $\mathrm{P}<0.05$ was accepted as significant statistically.

\section{Data Availability Statement}

Te datasets generated during and/or analyzed during the current study are available from the corresponding author on reasonable request.

\section{Ethical Approval and Informed Consent}

We confirm that all experimental protocols were approved by Qingdao Medical University (License number:17856321). The methods were carried out in accordance with the relevant guidelines and regulations; Informed consent was obtained from all participants and their legal guardians.

\section{Competing Interests Statement}

The authors declare that they have no conflict of interest with the contents of this article.

\section{Author Contributions}

Shulin Chen and Nailong Yang designed the study and revised the manuscript. Qiuling Wang, Weiwei Xiao, Guiqin Gu, Lili Xu, Dong Xing and Jinhuan Wang conducted most of the experiments. Qiuling Wang, Weiwei Xiao and Lili Xu wrote the paper. Qiuling Wang, Huafeng Li, Daping Wang and Lili Xu analyzed and interpreted the data. All authors reviewed and approved the manuscript.

\section{Funding}

The study was funded by Medicine Science and Technology Development Plan of Shandong Province (2013ws0037).

\section{References}

1. El Maghraoui A, Ouzzif Z, Mounach A, Ben Ghabrit A, Achemlal L, et al. (2011) The relationship between sex steroids, bone turnover and vertebral fracture prevalence in asymptomatic men. Bone 49(4): 853857.

2. Brand P, Khan A, Maduk C, Boyd S (2016) Are Fabry disease patients at risk of osteoporosis?.Mol Genet Metab 117(2): s30.

3. Legroux Géro I, Vignau J, Herbomez M, Flipo RM, Cortet B (2012) Predictive factors of change in BMD at 1 and 2 years in women with anorexia nervosa: a study of 146 cases. Osteoporos Int 23(12): 28552861.

4. Genant HK, Grampp S, Gluer CC, Faulkner KG, Jergas M, et al. (1994) Universal standardization for dual X-ray absorptiometry: Patient and phantom cross-calibration results. J Bone Miner Res 9(10): 1503-1514.

5. Zhu XY, Zhu HM, Zhang XM (2005) Comparison of accuracy and precision between two dual-energy X-ray absorptiometers. Chin J Osteoporosis 11(2): 189-194.

6. Yang NL, Yan SL, QN Wang J, Zhang LY (2006) Establishment of bone mineral density reference database of multiple skeletal regions in adult women in Qingdao area and its prediction for fracture risk. Chinese journal of endocrinology and metabolism 22(3): 209-212.

7. Kanis JA (1994) Assessment of fracture risk and its application to screening for postmenopausal osteoporosis: Synopsis of a WHO report. Osteoporosis int 4(6): 368-381.

8. Maraka S, Kennel KA (2015) Bisphosphonates for the prevention and treatment of osteoporosis. BMJ 3: 27-31.

9. Papaefthymiou MA, Bakoula C, Sarra A, Papassotiriou I, Chrousos GP, et al. (2014) Influence of hormonal parameters, bone mineral density and bone turnover on fracture risk in healthy male adolescents: a case control study. J Pediatr Endocr Met 27(7-8): 685-692.

10. Cortet B, Modi A, Tang J, Fan CP, Sajjan S, et al. (2016) Association between gastrointestinal events and osteoporosis treatment initiation in women diagnosed with osteoporosis in France: a retrospective analysis. BMC Musculoskelet Disord 17:195.

11. Arazi H, Ehsan E, Tahmineh S, Roya M (2016) The Relationship of Physical Activity and Anthropometric and Physiological Characteristics to Bone Mineral Density in Postmenopausal Women. J Clin Densitom 19(3): 382-388.

12. Jabbar S, Drury J, Fordham JN, Datta HK, Francis RM, et al. (2011) Osteoprotegerin, RANKL and bone turnover in postmenopausal osteoporosis. Journal of Clinical Pathology 64(4): 354-357. 
13. Bai MH, Ge BF, Bai J, Chen KM, Zhong R, et al. (2008) Analysis of bone mineral density in normal people in Lanzhou area, Chin J Osteoporosis 14: 736-737.

14. Wang WZ, Ma JF, Yang DZ, Jiang JJ, Shang JY, et al. (2000) Investigation of bone mineral density in middle-aged and aged people in Chengdu. Chin J Osteoporosis 6: 40-43.

\section{ISSN: 2574-1241}

DOI: 10.26717/BJSTR.2019.18.003129

Qingjie Xie, Daping Wang. Biomed J Sci \& Tech Res

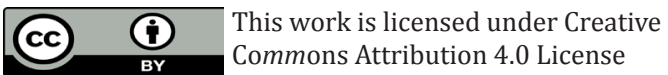

Submission Link: https://biomedres.us/submit-manuscript.php
15. Zhang ZH, Zhang ZR, Liu ZH, Yuan Wei (2016) A retrospective study of osteoporosis prevalence in mainland China using-2.0 SD as diagnosis criteria. Chin J Osteoporosis 21: 1-8.

16. (2011) Chinese society of osteoporosis and bone mineral research. The guidance of diagnosis and treatment of primary osteoporosis. Chin J Osteoporosis Bone Miner Res 4: 2-17.

$\begin{array}{ll}\text { BIOMEDICAL } & \text { Assets of Publishing with us } \\ \text { RESEARCHES } & \text { - Global archiving of articles } \\ & \text { - Immediate, unrestricted online access } \\ \end{array}$

